\title{
editorial
}

\section{Familiar faces}

W

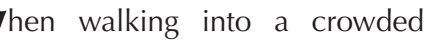
room, we inevitably look for familiar faces; an acquaintance in a room full of strangers is often the starting point for further social interactions or introductions, and can facilitate small talk. Seeking out the familiar is a standard human reaction to a new environment, whether it is a busy conference or an unexplored wilderness: the familiar is safe and, usually, reliable. Thus, the observation that it always seems to be the same people who speak at major meetings is due, in part, to the fact that organizers are more prone to ask wellknown 'club members', rather than risk inviting completely unknown people.

This 'inclusion barrier' has inevitable social consequences, but, more seriously, it can also influence research-sometimes with rather negative consequences. The feeling of being a little lost when walking into a crowded room is similar to the feeling of looking at a computer printout of a transcriptome or a protein network analysis. It contains all the results that have been sought—which genes are expressed after a specific treatment-but there is no Eureka! feeling because there are too many options: a buffet rather than a defined menu. Faced with this onslaught of information, many scientists feel overwhelmed and their obvious-and normal-reaction is to look for 'familiar faces': genes or proteins with easily recognizable names, rather than a numerical code.

As in a social context, these 'acquaintances' might not necessarily be the most interesting or relevant ones, but at least they come with some contextual information and some previous understanding. Like finding the edges of a jigsaw puzzle-never the most interesting or exciting parts_familiar pieces of information become the starting point for the complete picture. Eventually, of course, more pieces are added and more insights are gained, but we often approach a problem using the aspects with which we are familiar, instead of tackling the most challenging or interesting questions first.

This focus on recognizable things has its roots in the history of biological research. For decades, technology-or a lack thereof-limited the scope of molecular biological research to the extent that each group concentrated their work on one or a few individual genes-thus ignoring the potential roles that others might have. This is a self-perpetuating process: the increasing information available about these few select genes and their products means that their perceived importance grows and facilitates further research into them. Even today, with the enormously powerful tools of genomics and proteomics at hand, we still tend to focus our interest on the familiar, perhaps to the exclusion of other, more interesting, players.

Which brings us back to the familiar faces we have: why do some genes have names and others only have a number? Again, we must look to history. When biochemists started to map the enzymatic landscape of a cell, they concentrated on the metabolism of sugars. The relatively slow pace of this analysis allowed them to catalogue and name each gene. Their focus eventually extended to the cellular machinery of DNA replication and transcription, which created a framework into which new information from mutant studies could be added. But with the advent of new sequencing and high-throughput technologies, the number of genes and proteins known has exploded — so fast that many of these have only been assigned a number, as their function remains undefined.

The limits of this focus are becoming apparent, and never more so than when a knock-out study_in which a seemingly crucial gene is removed-does not stop the organism or cell from functioning. Under these circumstances, nature is usually able to fall back on redundant pathways, yet many of the alternative genes and proteins invoked remain unknown or are hidden behind an unfamiliar number. We are only just beginning to appreciate the combinatorial nature of physiological events and it seems likely that the combination of many proteins is as important as the absence or presence of a single protein.

Worse still, a name can even point us in the wrong direction. When subsequent studies show that the actual function of one factor in an extended protein network is completely unrelated to its original job description, its name does not change to reflect this new function. When an enzymatic function associated with a protein is subsequently shown to be a secondary activity, the protein still does not change its name. What is needed, in fact, is a scientific approach based on information rather than names. If all proteins and genes were number-coded, it would force scientists to focus on function rather than familiarity. Even if this were not possible, a more widespread use of bioinformatics and simple quantitative measures to prioritize the genes and other factors in a metabolic or regulatory network would, inevitably, lead to new perspectives and a focus on new, uncharacterized proteins. However comforting we might find the familiar, it has got us stuck in a rut laid down by chance and unrelated discoveries of proteins with now familiar faces; it might be time to make new friends.

\section{Frank Gannon \\ This Editorial represents the personal views of Frank Gannon and not those of Science Foundation Ireland or the European Molecular Biology Organization. \\ doi:10.1038/embor.2008.141}

